\title{
Self-Administered Videotape Therapy for Families With Conduct- Problem Children: Comparison With Two Cost-Effective Treatments and a Control Group
}

\author{
Carolyn Webster-Stratton, Mary Kolpacoff, and Terri Hollinsworth \\ Department of Parent and Child Nursing, University of Washington
}

\begin{abstract}
Parents of 114 conduct-problem children, aged 3-8 years, were randomly assigned to one of four groups: an individually administered videotape modeling treatment (IVM), a group discussion videotape modeling treatment (GDVM), a group discussion treatment (GD), and a waiting-list control group. Compared with the control group, all three treatment groups of mothers reported signifcantly fewer child behavior problems, more prosocial behaviors, and less spanking. Fathers in the GDVM and IVM conditions and teachers of children whose parents were in the GDVM and GD conditions also reported significant reductions in tehavior problems compared with control subjects. Home visit data indicated that all treatment groups of mothers. fathers, and children exhibited significant behavioral changes. There were relatively few differences between treatment groups on most outcome measures, although the differences found consistently favored the GDVM treatment. However, cost effectiveness was the major advantage of the IVM treatment.
\end{abstract}

Childhood conduct problems are prevalent and far reaching. Approximately two thirds of all young children who are referred to mental health agencies are eventually labeled antisocial. oppositional, or conduct disordered. Moreover, the prevalence of such children is increasing and creates a need that far exceeds available personnel and resources (President's Commission on Mental Health, 1978). The need to help families with conduct-problem children is particularly important because studies have shown that these children are at an increased risk not only for being abused by their parents (Burgess \& Conger, 1978; Patterson, 1977: Reid, Taplin, \& Loeber, 1981) but also for developing psychiatric disabilities as adults. particularly those evidenced by juvenile delinquency and crime (Loeber, 1985; Patterson, 1974; Robins, 1978. 1981; Rutter \& Geller. 1983).

Over the past decade, many treatment programs have focused on training parents to be the therapists for their conductproblem children. Several excellent literature reviews (Berkowitz \& Graziano, 1972; Moreland, Schwebel, Beck, \& Wells. 1982) have indicated that conduct-problem children show significant improvements following parent-training programs.

This research was supported by National Institutes of Health Institute of Nursing Grant 5 R01 NR01075-03. The authors are grateful to a number of people who assisted in data collection and data management: Andrea Alpar, Joyce Aoyama. Ming Chen, Andra Fjone, Maxine Fookson, Don Goldstein. Lois Hancock, Doris Harkness, Pat Huckell, Pat Loschen, Leslie Lee, Kay Peters, Lill Wagner, and Anna Webster. Special appreciation goes to Mary Hammond for her statistical assistance and to Bob McMahon for his review of an earlier draft of this article. Finally, thanks are extended to Barbara Hummel for careful preparation of the manuscript.

Correspondence concerning this article should be addressed to Carolyn Webster-Stratton, Department of Parent and Child Nursing, SC-74, University of Washington, Seattle. Washington 98195.
However. most parent-training approaches have been based on a traditional one-to-one treatment format with an individual therapist and, therefore, have been time-consuming, costly, and incapable of meeting increasing demands. Research is needed that focuses on more cost-effective parent-training interventions. Moreover, if one believes that families who can afford to obtain mental health services are not necessarily those most in need of services, one must also acknowledge a need to develop and investigate low-cost programs that have the potential for mass parent training in prevention and intervention.

One cost-effective approach to parent training is implemented through the use of standardized videotape modeling programs. The evaluation of videotape materials is in its infancy. Four studies (Flanagan, Adams, \& Forehand, 1979: Nay, 1976; O'Dell, Mahoney, Horton, \& Turner, 1979; O'Dell et al., 1982) have demonstrated that videotape modeling is superior to written materials. lectures, live modeling, or rehearsal. However, these studies have been limited by their emphasis on a single target parent behavior, such as time out or praise, rather than on a wider range of outcomes, such as child behavior changes or parent-child interactions. Webster-Stratton (1981a, 1981b, $1982 \mathrm{a}, 1982 \mathrm{~b}, 1984$ ) has reported the short- and long-term effectiveness of videotape modeling with clinic and nonclinic parent discussion groups for changing a broad range of parentchild interactions. Moreover, the group discussion videotape modeling treatment (GDVM) was shown to be as effective as a high-cost, one-to-one parent training approach for parents with conduct-problem children (Webster-Stratton, 1984). These studies have been limited by their inability to determine the critical ingredient of the GDVM treatment. It has not been known whether the videotapes, the group discussion, the therapist, or some combination of all three is the active component. If the videotape program does not need therapist-led group discussion to be effective, the program could be completely selfadministered and, therefore, widely disseminated at a low cost. 
The purpose of this randomized trial was to compare the outcomes of three cost-effective parent-training treatment programs in families with conduct-problem children: (a) individually administered videotape modeling (IVM); (b) group discussion videotape modeling (GDVM); and (c) group discussion only (GD). In addition, a waiting-list control group was studied.

\section{Method}

\section{Subjects}

Criteria for study entry required that (a) the child be between 3-8 years old; (b) the child have no debilitating physical impairment, intellectual deficit, or history of psychosis and be receiving no treatment at the time of referral; (c) the primary referral problem be child misconduct that had been occurring for more than 6 months (e.g., noncompliance, aggression, oppositional behaviors); and (d) parents rate their children as having a clinically significant number of behavior problems according to the Eyberg Child Behavior Inventory (ECBI; Eyberg \& Ross, 1978).

The 114 multiproblem families entered into the trial were either selfrefer red $(43 \%)$ or professionally referred $(57 \%)$. Study children included 79 boys and 35 girls with a mean age of 4 years and 6 months. The mean number of behavior problems reported at pretreatment according to the ECBI was $21.3(S D=6.2)$, indicating that the children were clearly in the clinic range according to Eyberg and Ross (1978) (for nonclinic range, $M=6.8, S D=3.9$ ). Home observations prior to treatment confirmed the ECBI results, with the children exhibiting noncompliant behaviors at a rate of almost $I$ a minute ( 22 in $30 \mathrm{~min}$ ) and deviant behaviors at a rate of 1 every $2 \mathrm{~min}$.

Study parents included 104 biological mothers and 70 biological fathers, 10 adoptive mothers, and 10 adoptive or stepfathers. Of these, 79 (69.3\%) were married and 35 (30.7\%) were single ( 34 mothers, 1 father). The mean age of mothers was 32.8 years and of fathers was 35 . I years. Family social class, as determined by Hollingshead and Redlich's (1958) Two-Factor Index, yielded a wide range of social class: Class $5(n=17)$, Class $4(n=26)$, Class $3(n=33)$, Class $2(n=24)$, and Class $I(n=14)$. Yearly income ranged from welfare level $(n=21)$ to less than $\$ 28,999$ $(n=31)$ to above $\$ 29,000(n=62)$. Interviews indicated that $36(31.6 \%)$ of the mothers had experienced spouse abuse and that $45(39.5 \%)$ of the families reported alcoholism or drug abuse in the immediate family and $70(61.4 \%)$ reported it in the extended family. Twenty three (20.2\%) of the mothers and $19(23.7 \%)$ of the fathers reported that they were abused as children. Fifteen (13.1\%) of the mothers reported prior involvement with Child Protective Services. Thirty three (31.1\%) of the mothers reported some depression, above a score of 10 on the Beck Depression Inventory (Beck, 1982).

\section{Assessment}

The goal of all three treatment programs was to teach parents how to reduce their children's behavior problems, particularly aggression and noncompliance, and how to increase their children's prosocial behaviors. Subjects were evaluated before and 1 month after treatment by parent perceptions of child adjustment, by mother biweekly observations and reports of discipline used, by parenting stress level, and by independent observations of parent-child interactions in the home. To assess treatment generalizability to other settings, teachers reported on the children's adjustment at school. Finally, there was a parent consumer-satisfaction measure concerning the therapy they received.

\section{Parent Perception of Child Adjustment}

Child Behavior Checklist (CBCL). The parent form of the CBCL (Achenbach \& Edelbrock, 1983) consists of 118 items, each rated on a 0-2-point scale. The items constitute multiple behavior-problem scales derived separately for boys and girls in different age groups (e.g., 4-5 years, 6-11 years). In this study, the Total Behavior Problem summary score was of primary interest because it applies to a variety of behavioral problems in all age and sex groups. The 8 girls and 16 boys under 4 years of age were scored on the CBCL form for 4-year-olds because they had reached that age by the time of posttreatment assessments.

Eyberg Child Behavior Inventory (ECBI). The ECBI (Eyberg \& Ross, 1978; Robinson, Eyberg, \& Ross, 1980) is a 36-item behavioral inventory of child conduct-problem behavior for 2-16-year-old children. The response format yields two scores: A Total Problem score, which indicates the total number of behavior problems, and an Intensity score, which indicates the frequency with which conduct problems occur. Reliability coefficients for the ECBI scales range from 0.86 (test-retest) to 0.98 (internal consistency).

\section{Mother Observations}

The Parent Daily Reports (PDR; Chamberlain, 1980; Chamberlain \& Reid, 1987) consist of a list of 19 negative and 19 prosocial behaviors commonly exhibited by children. During the intake, parents were asked to select those negative behaviors from the list that they felt were major problems as well as those positive behaviors that would be particularly pleasing to them if performed by their child. These individually tailored checklists were used as the basis for the phone calls that were conducted biweekly from the time of intake until the posttreatment assessment. During phone calls, the checklist was read to the mothers, who were asked to observe and report on the occurrence or nonoccurrence of the target behaviors for the previous $24 \mathrm{hr}$. After asking about the positive and negative behaviors on the PDR, the interviewer asked about the occurrence of spanking, time out, and low-rate events such as fire setting and running away. The PDR has been shown to have good test-retest reliability and to correlate significantly with concurrent home observation data (Chamberlain \& Reid, 1987).

\section{Parent Personal Adjustment}

The Parenting S1ress Index (PSI; Abidin, 1983) contains 126 items that are divided into two major domains reflecting stress in the parentchild relationship. The first domain represents parent characteristics and includes seven subscales that constitute the total Parent Domain score. These are Depression, Attachment, Restricted Role, Competence, Isolation, Spouse Support, and Health. The second domain, representing child characteristics. was not used in this study because there were already two other measures of child characteristics. The PSI has been shown by the authors to have acceptable content, concurrent, and construct validity. Alpha reliability coefficients were reported to be .95 , and test-retest reliabilities ranged from .82 to .71 (Abidin, 1983).

\section{Home Observations}

The Dyadic Parent-Child Interaction Coding System (DPICS; Robinson \& Eyberg, 1981), consisting of 29 behavior categories, was used to observe parent-child interactions in the home. From the parent behavior categories, three separate summary variables were formed: total praise, total critical statements, total no-opportunity commands (commands to which the child is given no opportunity to respond). For the target child, one variable was examined: total child deviance (the sum of the frequency of whine, cry, physical negative, smart talk, yell, destructive, and noncompliance ratings). In addition, a new nonverbal affect dimension developed by the authors was added, parent affect, which was defined as the emotional tone of the behaviors and was coded on the basis of nonverbal gestures, body posture, facial expressions, and tone of voice or inflections. Every $5 \mathrm{~min}$, coders paused and rated parent affect on a 5-point scale ranging from unrestrained negative affect (5) to 
neutral affect (3) to exuberant affect (1). These behaviors were selected from the DPICS coding system to focus on behaviors that have been shown to discriminate clinic from nonclinic families (Forehand. King, Reid. \& Yoder, 1975; Lobitz \& Johnson, 1975: Webster-Stratton, 1985).

Home observations were made by eight extensively trained observers who were blind to the hypotheses and to the group membership of the subjects. Initially, the observers received extensive training and were required to maintain $80 \%$ reliability with practice tapes before conducting home observations. It took approximately 4-6 months for observers to become reliable. To maintain accuracy, observers had weekly training sessions and practiced on videotaped interactions. To assess reliability, a second observer was present for at least $30^{\circ} \circ$ of all the home observations. Reliability was calculated in two ways: by the ratio of percent of agreements to total number of agreements and disagreements and by Pearson product-moment correlations between ratings for each separate behavior dimension. The percent agreement reliability was calculated for each 5-min segment and was based only on occurrences (not nonoccurrences) of behavior noted. Mean overall interrater agreement was $79 \%$ (range $=71-89 \%$ ) and the product-moment cor relations calculated between observers ranged from .70 (for affect) to .97 (for commands and praise).

\section{Teacher Perceptions of Child Adjustment}

The Behar Preschool Behavior Questionnaire (PBQ: Behar, 1977) includes 30 items. each rated on a 0-2-point scale, and is completed by the teachers of children between the ages of 3-7 years. Test-retest reliability has ranged from .60 to .99 . Factor analyses have yielded three subscales in addition to a Total Behavior Problem scale. The Total Behavior Problem scale was selected because it samples a broad range of conduct problems.

\section{Social Validity Measures}

The Consumer Satisfaction Questionnaire, which we adapted from the work of Forehand and McMahon (198I), consists of 40 items with a 7-point Likert scale response format. Statements were made to which the parent could respond (from strongly agree to strongly disagree). Responses were transformed into scores from 1 to 7 , with 7 the most positive. Four subscales measured parents' perceptions about child behavior improvement, format of treatment difficulty (e.g., videotapes, group discussion), treatment usefulness, and the overall difficulty of the parenting skills that were taught. The internal consistency of the subscales in this study ranged from .71 to .90 .

\section{Treatment Conditions}

Once subjects were accepted for entry, a randomly selected sealed envelope was opened that designated each family's parent-1raining condition. Families were continuously assigned at random to one of four conditions.

Group discussion videotape modeling training. The parents (28 mothers and 20 fathers) assigned to the GDVM condition came to the clinic weekly for 10-12 two-hour sessions. Each week, groups of 10-15 parents met with a therapist who showed them I of the 10 videotape programs of modeled parenting skills (approximately 250 vignettes and $25 \mathrm{~min}$ of videotape per program). After each presentation of a 2-min parent-child vignette, the therapist led a focused discussion of the important interactions and elicted parents' reactions, ideas. and questions about the material. A more complete description of the 10 videotape training programs and conceptual bases is available (Webster-Stratton. (981b, 1987).

Individually administered videotape modeling training. The parents ( 29 mothers and 20 fathers) assigned to the IVM condition came to the clinic weekly for 10-12 self-administered sessions. Single parents came alone and married parents usually came with their spouses. Each week a secretary provided them with a room and with 1 of the 10 videotape programs to watch. Parents were encouraged to pace themselves, to take as long as they needed to review a tape, and to review a tape a second time if necessary. On the average, weekly sessions lasted I hr. The IVM parents saw the same videotapes as the GDVM parents but did not receive the benefit of therapist feedback and therapist-led group discussion.

Group discussion training. The parents ( 28 mothers and 19 fathers) assigned to the GD condition came to the clinic weekly for 10-12 twohour sessions. They met in groups of $10-15$ parents with a therapist who led a group discussion of the same topics covered in GDVM. The only difference between this training and GDVM training was GDVM use of videotapes to illustrate content.

Waiting-list control group. The parents ( 29 mothers and 21 fathers) assigned to the control condition received no treatment and had no contact with a therapist. As with the other three treatment conditions, parents did receive biweekly PDR telephone calls concerning target child behaviors. The callers were warm, supportive, and reflective, but they did not offer any direct advice. After waiting 12 weeks, control subjects were assessed a second time and were then randomly assigned to one of the treatment conditions.

Training content, sequencing, and duration. Although the training methods differed for the three conditions, the content. sequencing, and number of treatment sessions was held constant. The content of all three training programs included a modification of the interactional model (Hanf \& Kling, 1973; Kogan \& Gordon, 1975), focusing on play skills, praises, and tangible rewards (Weeks 1-5). The last half of the program focused on teaching parents nonpunitive discipline approaches and a specific set of operant techniques (Patterson, 1975; Forehand \& McMahon, 198I) and problem-solving approaches (Spivack, Platt, \& Shure, 1976). Each of the three treatment programs had a minimum of 10 and a maximum of 12 weekly sessions.

\section{Therapists}

Two female clinicians and a female supervisor (ages $=39-45$ years) strved as therapists. Two were psychologists and one was a social worker. All therapists had children of their own and extensive prior experience (5-15 years) with behavior-problem children and family counseling. They received an intensive training program to learn the content and techniques of group therapy and videotape modeling. The therapy approaches for each of the treatment sessions were explicitly detailed in 10 treatment manuals and videotapes. Therapist training utilized live modeling by the supervisor, role playing, videotape feedback, and weekly group meetings. Therapist supervision was ongoing and consisted of live observation of groups, review of videotapes of group sessions, and weekly meetings to discuss cases and group dynamics. Each of the therapists was assigned an equal number of the GDVM and GD treatment groups to control for therapist effects.

\section{Treatment Integrity}

To assure the integrity of treatment, (a) therapists co-led their first group with the supervisor; (b) therapists followed a treatment manual for each session; (c) therapists kept detailed notes of each session documenting group process, the duration of the session, and parents' reactions; (d) all therapist-led group treatments were videotaped for feedback; and (e) therapists received ongoing supervision, feedback, and training throughout the study.

\section{Attrition and Attendance}

Of the 85 mothers and 59 fathers assigned to one of the three treatment groups, 78 mothers $\left(91.7^{\circ}\right)$ and 52 fathers $(88.1 \%)$ completed 
greater than $50 \%$ of the treatment sessions and the posttreatment assessments. Attendance indicated that GDVM mothers attended a mean of 10.1 sessions and fathers attended a mean of 9.1 sessions, that GD mothers attended a mean of 8.2 sessions and GD fathers attended a mean of 6.3 sessions, and that IVM mothers attended a mean of 9.6 sessions and fathers attended a mean of 9.2 sessions. Significantly $(p<.01)$ more mothers and fathers missed weekly sessions and dropped out from the GD treatment compared with the GDVM and IVM treatments.

\section{Procedure}

When possible, fathers as well as mothers completed the parent report measures (CBCL, ECBI, PSI), but instructions were given to complete the questionnaires separately. Due to practical limitations, only the mothers (with the exception of one single father) received the biweekly telephone calls (PDR). For those children in daycare or school $(n=84)$, the PBQ was sent to teachers to complete. The teachers were told not that the children had behavior problems but rather that the parents were participating in a child development study.

Each mother-child or father-child interaction was observed in the home for $30 \mathrm{~min}$ on two evenings during the week (between 4:30 p.m. and 7:30 p.m.). During these observations, an attempt was made to impose as little structure as possible. and family members were asked to "do what you would normally do" except talk to the observers, watch television, or talk on the telephone. Whether the mother or father was observed first was randomly determined.

Families were admitted to the study in four cohorts. The first cohort of 28 families completed baseline assessments, and each family was randomly assigned to one of the four conditions. Following completion of the treatment programs, all four groups were reassessed on the same measures. As the first cohort neared completion, the second cohort of 28 families received baseline assessment and was randomly assigned to the four conditions. This process was continued for four identical phases.

\section{Results}

Treatment effects were evaluated by mother and father selfreport measures (ECBI, CBCL, PSI); by maternal daily observations and reports of discipline (PDR); by mother and father behaviors (no-opportunity commands, praise, criticisms, affect); by child behaviors (total deviance): by teacher reports (PBQ); and by parent consumer satisfaction. With baseline values serving as the covariates. each set of dependent measures was initially submitted to a multivariate analysis of covariance (MANCOVA). When the MANCOVA revealed a significant effect, a four-group analysis of covariance (ANCOVA) was performed for each of the dependent variables. This was followed by preplanned comparisons of each treatment group with the control group and with each other. For each set of dependent variables, the Dunn-Bonferonni tables were used to determine the critical values in order to correct for the number of individual comparisons.

The multivariate analyses of variance (MANOVAs) revealed no significant differences between the four groups on the demographic or family background variables (marital status, education, income, social class, or child's sex and age).

\section{Mother Report Measures}

The MANCOVA revealed a significant group effect for the set of four mother report variables. $F(12,249)=4.68, p<.001$.

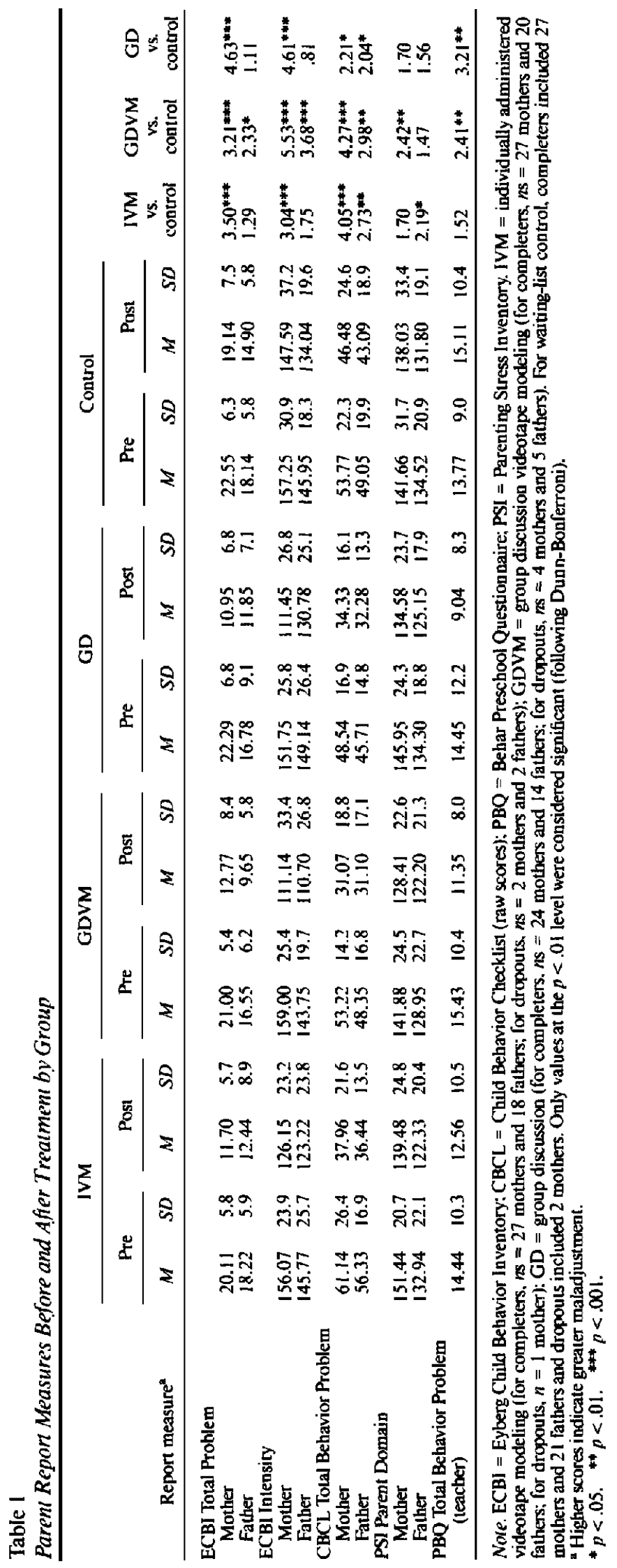


Significant differences for each of the four mother-report variables were also reported by the four-group ANCOVA. Table 1 presents mean scores, standard deviations, and $t$ values for the mother-report variables. For the IVM treatment, all three of the mother reports of children's behaviors significantly improved in the predicted directions. Only the PSI Parent Domain score did not show a significant difference. For the GDVM treatment, all four of the mother reports significantly improved compared with those from control mothers. For the GD treatment, two of the mother reports significantly improved in the predicted directions. When the three treatment groups were compared with each other, they were markedly similar. The only significant difference was that GDVM mothers reported a lower ECBI Intensity score than did IVM mothers, $l(100)=2.50, p<.01$.

\section{Mother Observations}

The MANCOVA revealed a significant group effect for mother observations and reports on the PDR variables, $F(15,249)=$ $3.40, p<.001$. Table 2 presents mean scores, standard deviations, and $t$ values for these variables. Only values at the .01 level were considered significant according to the Dunn-Bonferroni tables. For the IVM treatment, biweekly telephone reports indicated that, compared with control mothers' observations, IVM mothers observed significantly fewer negative behaviors and more prosocial behaviors in their children but observed no change in low-rate events (running away, fire setting, destruction). They also reported a significant decrease in their use of time out and spanking. The GDVM mothers observed signifcantly fewer target negative behaviors and low-rate events and a trend $(p<.05)$ toward increased prosocial behaviors in their children. They also reported a significant reduction in time out and spanking. The GD mothers reported similar significant differences (except for time out use) compared with control mothers. When the three treatment groups were compared with each other, the only significant difference was that GDVM mothers observed fewer target negative behaviors than GD mothers, $t(100)=2.50, p<.01$.

\section{Father Report Measures}

The MANCOva revealed a significant group effect for the set of four father report variables, $F(12,162)=1.87, p<.05$. A four-group ANCOVA revealed significant differences on two of the four father variables. Table 1 presents mean scores, standard deviations, and $t$ values for the father-report variables. For the IVM treatment, fathers reported significantly lower scores on the CBCL and a trend $(p<.05)$ for a reduction in the PSI Parent Domain score. The GDVM fathers reported significantly lower scores on the CBCL and the ECBI Intensity score and a strong trend $(p<.02)$ for a reduction in the ECBI Problem score. The GD fathers did not report significant differences on any of the report variables, although there was a trend $(p<.05)$ for fewer behavior problems on the CBCL. When the three treatment groups were compared with each other, the only significant difference was that GDVM fathers reported a lower ECBI Intensity score than did GD fathers, $t(68)=2.49, p<.01$.

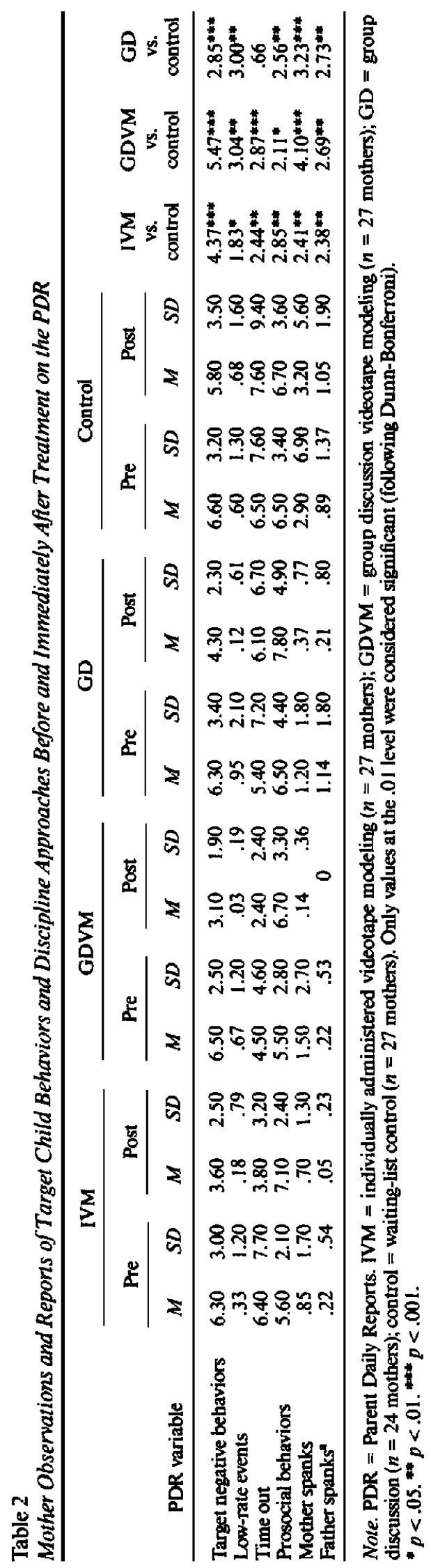




\section{Teacher Reports}

A four-group ANCOVA revealed a significant overall group effect on the PBQ Total Behavior Problem score $F(3,80)=3.80$, $p<.01$. Further analyses indicated that the teachers of children in the GDVM and GD conditions reported significant reductions in children's behavior problems compared with teachers of children in the control group. When the three treatment groups were compared with each other, there were no signifcant differences.

\section{Mother Behavior Summary V'ariables}

The MANCOVA revealed a significant group effect for the set of four mother behavior variables, $F(12.209)=1.94, p<.03$. There were significant differences on all four of the variables by four-group ANCOVA. Table 3 presents mean scores. standard deviations, and $t$ values for the mother-behavior variables. For the IVM treatment, two of the four mother behaviors showed a significant difference compared with control mothers' behaviors, and there was also a strong trend $(p<.05)$ for a reduction in no-opportunity commands. For the GDVM treatment, all four of the mother behaviors showed significant differences in the predicted directions compared with control mothers' behaviors. For the GD treatment, three of the mother behaviors showed significant differences compared with those of control mothers. The three treatment groups were again comparable on all mother-behavior variables, with the single exception that the GDVM mothers exhibited significantly more praise than IVM mothers, $t(100)=2.61, p<.01$.

\section{Father Behavior Summary Variables}

The MANCOVA revealed a significant group effect for the set of father-behavior variables $F(12,114)=2.07, p<.02$. There were significant differences on all four of the variables by fourgroup ANCOVA. Table 3 presents mean scores, standard deviations, and $t$ values for the father-behavior variables. For the IVM treatment, two of the four father behaviors showed a significant difference compared with control fathers' behaviors. For the GDVM treatment, two of the father behaviors showed significant differences and the other two behavior variables showed trends $(p<.06)$ in the predicted directions. For the GD treatment, one of the behavior variables showed a significant difference, and there was also a trend $(p<.05)$ for two other behavior variables to be reduced as predicted. When the treatment groups were compared with each other, there was only a single significant difference, with the GDVM fathers exhibiting significantly more praise statements than the GD fathers, $l(68)=$ $2.76, p<.01$.

\section{Child Behavior Variables}

A four-group ANCOVA revealed a significant overall group effect for total child deviance when children were observed interacting with mothers, $F(3,96)=2.58, p<.05$. The GDVM and GD children showed significant reductions in total deviance compared with control children, whereas IVM children showed a trend in the predicted direction. When the children of the three treatment groups were compared with each other,

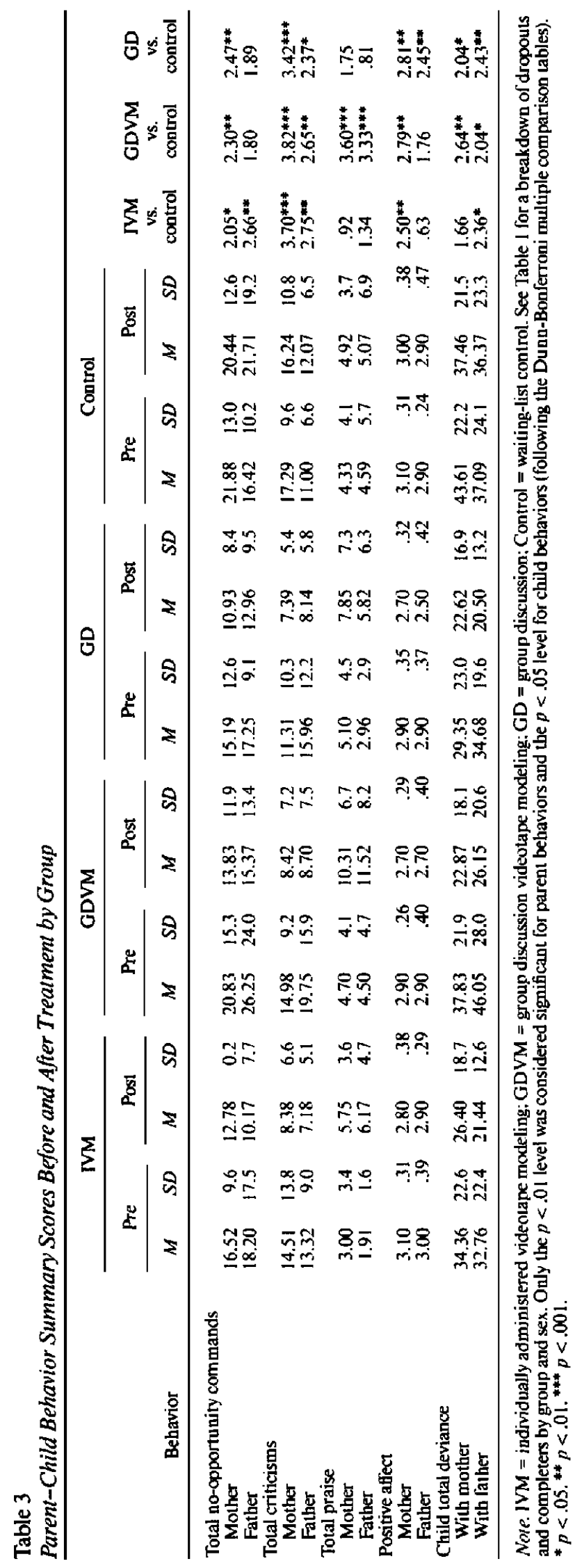


Table 4

Consumer Satisfaction Measure Results Immediately After Treatment

\begin{tabular}{|c|c|c|c|c|c|c|c|c|c|}
\hline Consumer satisfaction subscale & $M$ & $S D$ & $M$ & $S D$ & $M$ & $S D$ & $\begin{array}{l}\text { GDVM } \\
\text { vs. } \\
\text { GD }\end{array}$ & $\begin{array}{l}\text { GDVM } \\
\text { vs. } \\
\text { IVM } \\
\end{array}$ & $\begin{array}{c}\text { GD } \\
\text { vs. } \\
\text { IVM } \\
\end{array}$ \\
\hline \multicolumn{10}{|l|}{ Behavior improvement posttreatmen $t^{\mathrm{A}}$} \\
\hline Mother & 64.96 & 5.2 & 68.37 & 5.3 & 64.87 & 6.6 & $2.18^{*}$ & $2.19^{*}$ & .05 \\
\hline Father & 64.22 & 5.0 & 67.28 & 4.0 & 66.76 & 5.9 & .30 & $1.93^{*}$ & 1.41 \\
\hline \multicolumn{10}{|l|}{ Format of treatment difficulty ${ }^{b}$} \\
\hline Mother & 24.81 & 2.9 & 27.29 & 3.7 & 27.29 & 3.8 & .005 & $2.62^{* *}$ & $2.62^{* * *}$ \\
\hline Mother & 29.48 & 3.0 & 31.52 & 2.5 & 31.67 & 2.3 & .20 & $2.86^{* *}$ & $3.00^{* *}$ \\
\hline Father & 27.50 & 3.6 & 29.66 & 2.4 & 30.46 & 2.7 & .75 & $2.25^{*}$ & $2.71^{* *}$ \\
\hline \multicolumn{10}{|l|}{ Overall difficulty ${ }^{c}$} \\
\hline Mother & 5.07 & .67 & 5.52 & 1.0 & 4.79 & 1.1 & $2.71^{* * *}$ & 1.70 & 1.05 \\
\hline Father & 5.11 & .90 & 5.23 & .83 & 5.00 & 1.2 & .70 & .41 & .32 \\
\hline
\end{tabular}

- Score range $=11-77 .{ }^{b}$ Score range $=5-35 .{ }^{c}$ Score range $=1-7$.

$* p<.05{ }^{* *} p<.01{ }^{* * *} p<.001$.

there were no significant differences in their interactions with their mothers.

An ANCOVA also revealed a significant group effect for total child deviance when children were interacting with their fathers, $F(3,67)=2.84, p<.04$. Children from all three treatment groups exhibited a significant drop in total child deviance when observed interacting with their fathers compared with control children's behaviors with their fathers. When the three treatment groups were compared with each other, there were again no significant differences (see Table 3).

\section{Consumer Satisfaction}

From 1 to 2 months after treatment, all three treatment groups reported high scores on the consumer satisfaction measure for treatment format usability, difficulty, and child improvements (see Table 4). A MANOVA revealed a significant overall group effect for the four mother consumer-satisfaction variables, $F(12,249)=2.69, p<.002$. Mothers in the GDVM treatment condition reported more satisfaction with child behavior improvements posttreatment than did GD and IVM mothers. They also found the content of the treatment techniques to be significantly easier to carry out than did the GD mothers and found the format of GDVM treatment to be more useful than did the IVM mothers. The GD mothers reported that their treatment was significantly more useful and easier to use than the IVM mothers. The MANOVA did not reveal a significant group effect for the fathers' consumer-satisfaction variables, $F(12,167)=1.11, p<.35$.

\section{Discussion}

The primary purpose of this study was to evaluate a new selfadministered videotape modeling treatment program in comparison with two other cost-effective and widely applicable programs of treatment for families of conduct-problem children. The first major finding was that, when compared with control mothers, all three treatment groups of mothers reported highly significant improvements in their children's behaviors on the
CBCL, the ECBI, and the PDR. The GDVM and IVM fathers also reported significantly fewer child behavior problems compared with control fathers. Observations indicated that, when compared with control parents, mothers and fathers from all three treatment groups exhibited significant interactional changes with their children on at least two of the four behavior variables. Moreover, children showed significant reductions in deviant behaviors when they interacted with fathers from all three treatment conditions and when they interacted with mothers from the GDVM and GD treatments.

The second purpose of the study was to compare differences hetween the three treatment programs to determine whether the active ingredient of treatment was group support and discussion, skilled therapist intervention, or videotape modeling. Overall, there were relatively few differences detected between the three programs on most outcome measures. However, differences consistently seemed to favor the combined GDVM treatment. Compared with the control condition, the GDVM condition was the only treatment that significantly reduced mothers' reports of parenting stress and fathers' reports of the intensity of child behavior problems and that increased both mothers' and fathers' praise statements. The significantly higher consumer satisfaction scores, lower dropout rate, and higher attendance also corroborated the trend for the GDVM treatment to be superior. These results replicate a previous study using GDVM (Webster-Stratton, 1984) and suggest that videotape modeling enhances the effectiveness of therapist-led group discussions.

However, of particular interest in this study were the findings relating to the IVM treatment. Analyses indicated that IVM was not quite as potent as GDVM but was at least comparable with GD treatment. Compared with control mothers, IVM mothers reported significant reductions in their children's behavior problems and were observed to be less critical and to have more positive affect while interacting with their children. Compared with control fathers, IVM fathers reported significant reductions in their children's behavior problems and were observed to give fewer commands and critical statements. In 
addition, children were observed to be less deviant in their interactions with IVM fathers than with control fathers. Moreover, only two mothers and fathers dropped out of this treatment, significantly fewer than the nine parents who dropped out from the GD treatment. These findings are rather remarkable because these multiproblem families with conduct-problem children had no direct therapist contact or group support throughout the entire training series. The findings suggest that parents are both motivated and capable of learning to change their own behaviors, as well as their children's behaviors, from a self-administered videotape program. Perhaps one of the reasons for the effectiveness of the standardized IVM program is that it enhances the parents' abilities to generalize by exposing them to over 250 vignettes of parents and children in a variety of problem situations. This variety and complexity of content may be harder to present in a group discussion format. Another possibility is that the IVM program increases self-efficacy by allowing families to solve problems and be responsible for their own treatment. The IVM program offers an opportunity for privacy, flexible scheduling, self-pacing, and self-control, which is also difficult to achieve in a group therapy approach. However, the major advantage of the IVM program compared with the other treatments was its cost effectiveness. This approach involved no therapist time, required half the weekly time commitment from parents than that required by the two group treatments ( $1 \mathrm{hr}$ vs. $2 \mathrm{hr}$ per week) and yet yielded close to equivalent results.

There are several limitations to the present study. First, the long-term effects of the three treatment programs are unknown. It may be postulated that the initial effects of IVM will not be maintained compared with those of the other two treatment approaches, which involved skilled therapist feedback and group support. Follow-up data are needed to fully understand the ability of these treatment programs to produce clinically relevant outcomes, such as whether the children continue to improve. Second, treatment effects were examined without consideration of factors related to specific treatment responders or nonresponders. It may be important to determine the characteristics of families who can be helped with a particular model of intervention. Nonetheless, these data suggest that videotape modeling programs may be a promising format for initial use in a variety of applications. They may be used to increase the effectiveness of parent-training groups, for preventive education programs, or for the initial phase of a treatment approach. Their cost effectiveness and ability to be disseminated to large numbers of people make them an appealing alternative to the more traditional forms of treatment for child behavior problems. Further research is now needed to enhance the effectiveness of self-administered videotape programs and to better target who can best benefit from such programs. In conclusion, Rosen recently reminded psychologists of their social responsibility to "learn how to help people help themselves." However, he cautioned that "self-help therapies need to be systematically developed and assessed in order to understand their risks as well as their benefits" (1987, p. 50).

\section{References}

Abidin, R. R. (1983). Parenting Stress Inder-Manual. Charlottesville, VA: Pediatric Psychology Press.
Achenbach, T. M., \& Edelbrock. C. S. (1983). Manual for the Child Behavior Checklist and Revised Child Behavior Profile. Burlington, VT. University Associates in Psychiatry.

Beck. A. T. (1982). Depression: Causes and treatment. Ann Arbor, MI: University of Michigan Press.

Behar, L. (1977). The preschool behavior questionnaire. Journal of $A b$ normal Child Psichology: 5, 265-275.

Berkowitz, B. P., \& Graziano, A. M. (1972). Training parents as behavior therapists: A review. Behavior Research and Therapy, 10, 297317.

Burgess, R., \& Conger, R. (1978). Family interactions in abusive, neglectful, and normal families. Child Development, 49, 1163-1173.

Chamberlain, P. (1980). A parent daily report measure. Unpublished doctoral dissertation, University of Oregon.

Chamberlain, P., \& Reid, J. B. (1987). Parent observation and report of child symptoms. Behavioral Assessment, 9, 97-109.

Eyberg, S. M., \& Ross, A. W. (1978). Assessment of child behavior problems: The validation of a new inventory. Journal of Clinical Psychology: $16,113-116$.

Flanagan, S., Adams. H. E., \& Forehand, R. (1979). A comparison of four instructional techniques for teaching parents to use time out. Behavior Therapy, 10, 94-102.

Forehand, R. L., King, H. E., Reid, S., \& Yoder, P. (1975). Motherchild interactions: Comparison of a noncompliant clinic group and a nonclinic group. Behavior Research and Therapy, 13, 79-84.

Forehand, R. L., \& McMahon, R. J. (1981). Helping the noncompliant child: A clinician's guide to parent training. New York: Guilford Press.

Hanf, C.. \& Kling, F. (1973). Facilitating parent-child interaction: $A$ two-stage training model. Unpublished manuscript, University of Oregon Medical School.

Hollingshead, A. B., \& Redlich, F. C. (1958). Social class and mental illness. New York: Wiley.

Kogan, K. L., \& Gordon, B. M. (1975). A mother-instruction program: Documenting change in mother-child interactions. Child Psychiatry' and Human Development, 5, 189-200.

Lobitz, G. K., \& Johnson. S. M. (1975). Normal versus deviant children: A multi-method comparison. Journal of Abnormal Child Psychology: 3, 353-374.

Loeber, R. (1985). Patterns and development of antisocial child behavior. In G. J. Whitehurst (Ed.), Annals of child development (Vol. 2, pp. 77- (16). New York: JAl Press.

Moreland, J. R.. Schwebel, A. I., Beck, S., \& Wells, R. T. (1982). Parents as therapists: A review of the behavior therapy parent training literature 1975 to 1981 . Behavior Modification, 6, 250-276.

Nay, R. W. (1976). A systematic comparison of instructional techniques for parents. Behavior Therapy; 6, 14-21.

O'Dell, S. L., Mahoney, N., Horton, W., \& Turner, P. (1979). Mediaassisted parent training: Alternative models. Behavior Therapy: 10 , 103-110.

O'Dell, S. L., O'Quinn, G. A., Alford, B. A., O'Briant, A. L., Bradlyn, A. S., \& Giebenhain. J. E. (1982). Predicting the acquisition of parenting skills via four training methods. Behavior Therapj; 13, 194208.

Patterson, G. R. (1975). Families: Applications of social learning to family' life. Champaign, IL: Research Press.

Patterson, G. R. (1977). A performance theory for coercive family interaction. In L. Cauns (Ed.), Social interaction: Methods, analyses, and illustrations (pp. 119-162). Chicago: University of Chicago Press.

Patterson, G. R. (1974). Interventions for boys with conduct problems: Multiple setting treatments and criteria. Journal of Consulting and Clinical Psychology: 42, 471-481.

President's Commission on Mental Health. (1978). Report to the President (Vols. 1-4). Washington, DC: U.S. Government Printing Office. 
Reid, J., Taplin, P., \& Loeber, R. ( 198 I ). A social interactional approach to the treatment of abusive families. In R. Stuart (Ed.), Violent beharior: Social learning approaches to prediction, management and treatment (pp. 83-101). New York: Brunner/Mazel.

Robins, L. N. (1978). Study predictors of adult antisocial behavior. $P s y$ chological Medicine, 8, 611-622.

Robins. L. N. (1981). Epidemiological approaches to natural history research: Antisocial disorders in children. Journal of the American Academy of Child Psychiatry, 20, 566-580.

Robinson, E. A.. \& Eyberg, S. M. (1981). The dyadic parent-child interaction coding system: Standardization and validation. Journal of Consulting and Clinical Psychology; 49, 245-250.

Robinson, E. A., Eyberg, S. M., \& Ross, A. W. (1980). The standardization of an inventory of child conduct problem behaviors. Journal of Clinical Child Psichology; 9, 22-28.

Rosen, G. M. ( 1987). Self-help treatment books and the commercialization of psychotherapy. American Psychologist, 42. 46-51.

Rutter, M., \& Geller, H. (1983). Juvenile delinquency: Trends and perspectives. New York: Penguin Books.

Spivack. G., Platt. J. J., \& Shure. M. B. (1976). The problem solving approach to adjustment. San Francisco: Jossey-Bass.

Webster-Stratton. C. (1981a). Modification of mothers' behaviors and attitudes through videotape modeling group discussion. Behavior Therapy, 12, 634-642.

Webster-Stratton, C. (1981b). Videotape modeling: A method of parent education. Journal of Clinical Child Psychology; 10, 93-97.

Webster-Stratton, C. (1982a). Long term effects of a videotape modeling parent education program: Comparison of immediate and I-year followup results. Behavior Therapy; 13, 702-714.

Webster-Stratton, C. (1982b). Teaching mothers through videotape modeling to change their children's behaviors. Journal of Pediatric Psychology! 7, 279-294.

Webster-Stratton, C. (1984). Randomized trial of two parent-training programs for families with conduct-disordered children. Journal of Consulting and Clinical Psychology: 52, 666-678.

Webster-Stratton, C. (1985). Mother perceptions and mother-child interactions: Comparison of a clinic-referred and a nonclinic group. Journal of Clinical Child Psychology: 14, 334-339.

Webster-Stratton, C. (1987). Parents and children: A 10 program videotape parent training series n'ith manuals. Eugene, OR: Castalia Press.

Received July 21, 1987

Revision received November 16, 1987 Accepted December 4, 1987 